\title{
3D TOPOLOGICAL INDOOR BUILDING MODELING INTEGRATED WITH OPEN STREET MAP
}

\author{
Ali Jamali ${ }^{\mathrm{a}}$, Alias Abdul Rahman a and Pawel Boguslawski ${ }^{\mathrm{b}}$ \\ ${ }^{a}$ Universiti Teknologi Malaysia (UTM), Faculty of Geoinformation and Real Estate \\ ali.jamali.65@gmail.com, alias@utm.my \\ ${ }^{\mathrm{b}}$ University of the West of England, Faculty of Environment and Technology. \\ Pawel.boguslawski@uwe.ac.uk
}

KEY WORDS: Indoor Surveying, 3D Data Modeling, Topology, 3D Indoor Building, Open Street Map

\begin{abstract}
:
Considering various fields of applications for building surveying and various demands, geometry representation of a building is the most crucial aspect of a building survey. The interiors of the buildings need to be described along with the relative locations of the rooms, corridors, doors and exits in many kinds of emergency response, such as fire, bombs, smoke, and pollution. Topological representation is a challenging task within the Geography Information Science (GIS) environment, as the data structures required to express these relationships are particularly difficult to develop. Even within the Computer Aided Design (CAD) community, the structures for expressing the relationships between adjacent building parts are complex and often incomplete. In this paper, an integration of 3D topological indoor building modeling in Dual Half Edge (DHE) data structure and outdoor navigation network from Open Street Map (OSM) is presented.
\end{abstract}

\section{INTRODUCTION}

According to Donath and Thurow (2007), considering various fields of applications for building surveying and various demands, geometry representation of a building is the most crucial aspect of a building survey. In the previous researches (Becker et al., 2009; Liu and Zlatanova, 2012), 3D modeling of buildings and their relationships with the surrounding roads and terrain were provided, but their utility is limited in many cases of navigation and emergency planning, as only the exteriors of the buildings may be analyzed. For many kinds of emergency response to fire, bombs, smoke, and pollution - the interiors of the buildings need to be described, along with the relative locations of the rooms, corridors, doors and exits.

Topological representation has been a difficult task within the GIS environment, as the data structures required to express these relationships, are particularly difficult to develop. Even within the Computer Aided Design (CAD) community, the structures for expressing the relationships between adjacent building parts are complex and often incomplete.

Unfortunately many methods used for land surveying cannot be easily applied because of, to name a few: lack of Global Positioning System (GPS) signal from satellites in indoor environment; limited working area inside buildings especially in office space; very detailed environment with furniture and installations. There are four approaches that seem to be suitable for indoor surveying. 1) Laser scanning (Dongzhen et al., 2009) which is expensive and complex; requiring considerable modeling effort to fit sections of the resulting point cloud to basic features such as walls, resulting in extensive manual work after data collection and no easy way to integrate individual scan results with the model of a complete complex building (Yusuf, 2007). 2) Traditional surveying with a Total Station or equivalent is also possible, but conversion of captured data points into a building model requires complex, and so far unavailable, software. 3) A light 'Laser Rangefinder' which integrates azimuth (from a digital compass) and inclination along with the laser rangefinder, although it has a lower level of accuracy than the Total Station (Jamali et al., 2015). 4) A photogrammetry technique uses un-calibrated non-metric cameras to extract 3D information from a scene of images. For indoor surveying it is as simple as taking pictures. Additionally images can be used for a texture extraction textures can be attached to walls, floors, and ceilings in the model which would increase the realism of visualization.

In this paper, 3D topological modeling of indoor building using DHE data structure is presented. This paper is organized as follows: Section 2 presents background of indoor building modeling. In section 3, for 3D building modeling DHE data structure is used. Data collection for navigation network outside the building using OSM is presented in section 4. Conclusion is discussed in section 5 .

\section{PREVIOUS WORKS}

According to Liu and Zlatanova (2011), an up to date and accurate navigation model to be considered is most crucial requirement for developing a robust emergency response system: semantically rich knowledge, automated generation 
of network, dynamic routing capacity and door-to-door movement capability are some of the requirements in indoor navigation modeling.

Semantically rich knowledge provides useful information about geometrical object such as door locations or exit points in a building. As shown in Figure1, Liu and Zlatanova (2012) explained door to door route as "the straight medial axis of a corridor is D1-M1-M2-M3-M4-D4. Even if the person could not see the door D3 from D1, the "door to door" route could be D1-S1-D3 (the dash line), which means the person will see D3 until he reaches $S 1$. Yet the dual graph route would be D1-M1-M2-M3-D3 in this case".

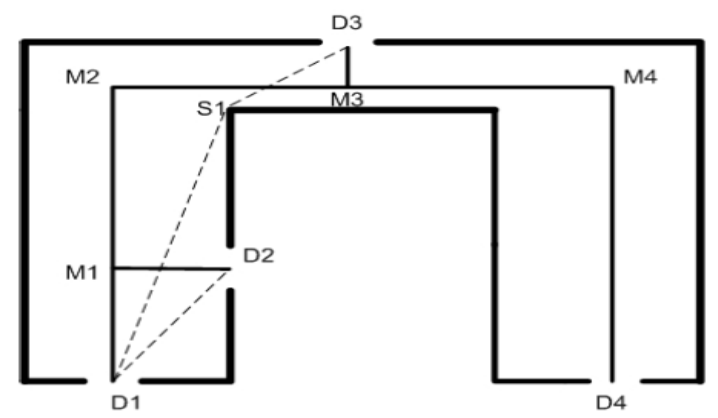

Figure 1. An example of door to door routes (Liu and Zlatanova, 2011).

Existing indoor navigation models as mentioned by Liu and Zlatanova (2011) are as follows:

Lee (2001) proposed conventional dual based on the Poincare Duality theory. For connectivity of building, conventional dual uses Node-Relation Structure (NRS). GNM was an extension of NRS to represent more accurate indoor building environment (Lee, 2004). For improving construction of GNM, a skeleton-abstraction algorithm was proposed by Lee (2004), which is based on the StraightMedial Axis Transformation (S-MAT). Becker et al. (2009) proposed a Multilayered Space-event which in their model each space layer is divided into primal and dual space with topology and geometry division of spaces.

Many researchers have used Conventional Dual Graph (Lee 2004; Li and He 2008; Becker et al. 2009; Boguslawski et al. 2011). To improve S-MAT, Yuan and Schneider (2010) proposed Direct Path Graph (DPG) which consists of cells, path segments and accessibilities.

Navigable space model (Slingsby and Raper 2008, Schaap 2010) is topologically-connected and navigable space (surfaces) which leads to pedestrian's movement. Navigable space model is a $2.5 \mathrm{D}$ reconstructed model of 2D plan with limited height and space constraints. Network generation in complex scenarios and automatic generation of network are challenging and there is no door-to-door movement in this model.

Yuan and Schneider (2010) proposed LEGO-graph which is a Regular-grid graph based on the 3D voxel; LEGOgraph using different width and heights can provide all possible paths. The Regular-grid model can be a 2D or 3D model. 3D Regular-grid model is an extension of 2D model. 3D model voxel could be used to represent indoor building structure. In Regular-grid graph nodes represent grid and edges of a specific node represent relationship between the node and its surrounding neighbors ( $\mathrm{Li}$ et al. 2009). Bandi and Thalmann (1998) discredited building parts into 3D voxel to compute an obstacle-free route with surmountable and insurmountable obstacle.

Considering several criteria (e.g. distance between walls, visibility) the Sub-division intends to divide $2 \mathrm{D}$ plans into cells. Lamarche and Donikian (2004) used constrained Delaunay triangulation algorithm and Convex Cell optimization to subdivide a 2D plan. Lorenz et al. (2006) divided a 2D plan into cells to create a graph structure. As shown by Figure 2, in their model Cell centers are connected to doors.

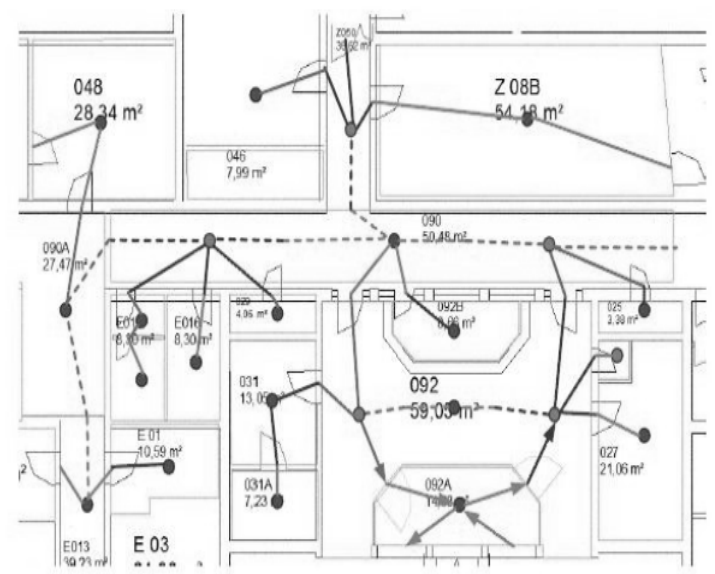

Figure 2. Cell centers and paths overlaid with a floor Plan (Lorenz et al. 2006).

Stoffel et al. (2007) used visibility criteria to divide a plan into cells. As can be seen in Figure 3, in their model, a plan is partitioned into non-overlapping convex sub-regions (cells) according to visibility conditions.

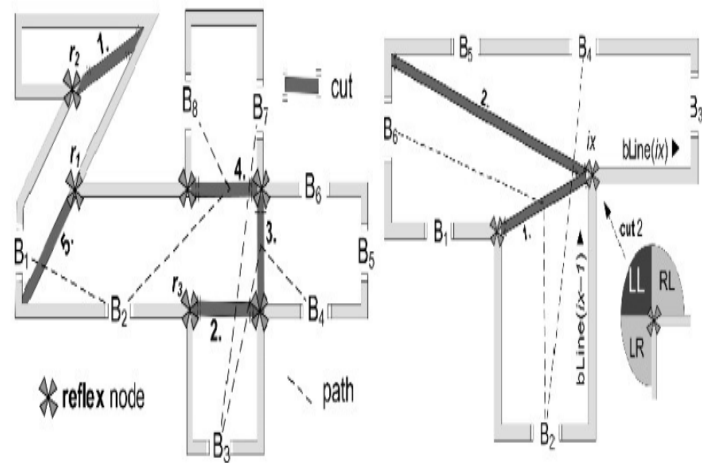

Figure 3.Visibility partitioning result (Stoffel et al. 2007).

According to Liu and Zlatanova (2011) a 3D sub-division with visibility consideration is more accurate and suitable 
approach for indoor navigation routing as shown by Figure 4. "Possible outlets encompass inner room doors (denoted by " $I ")$, room doors (denoted by " $D$ "), stairs (denoted by "S") and windows (denoted by "W"). Each opening is abstracted as a node of a network model (see in the bottomleft part of Figure 4). If two openings are mutually visible to each other, then there is an edge of the network between the two nodes. The dash-line edges in Figure 4 indicate windows will be used for escape only in some specific conditions (e.g. broken in an emergency situation)".

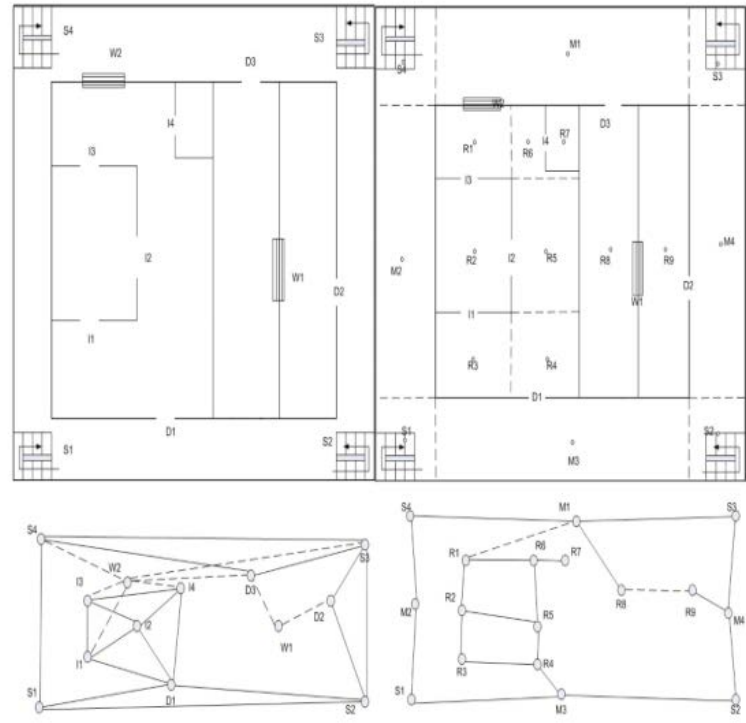

Figure 4. Indoor visibility structure and conventional dual graph model (Liu and Zlatanova, 2011).

Tashakkori et al. (2015) proposed a new technique for indoor building navigation. In their method three components including indoor building information, outdoor emergency information and dynamic and semantic building information has been addressed (See Figure 5).

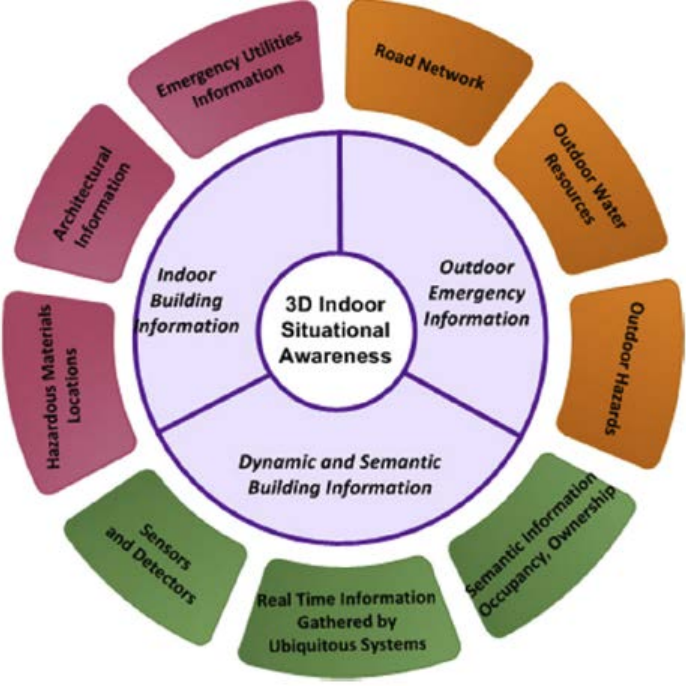

Figure 5. Dimensions of 3D indoor situational awareness (Tashakkori et al., 2015).
Tashakkori et al. (2015) in their proposed method of navigation defined three different scenarios including navigation assistance, situation awareness and indoor route blockage.

Karas et al. (2006) extracted navigation network from floor plans using MUSCLE-M technique (see Figure 6). There are two main aspects in Karas et al. (2006) research including 3D building modeling and navigation network modeling which topological model is extracted from floor plans (Topology is derived from geometrical model).

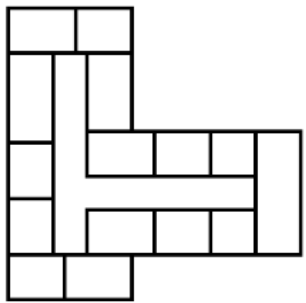

(a)

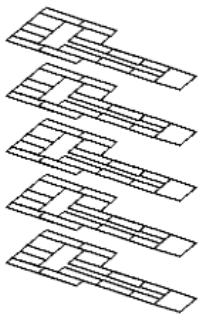

(c)

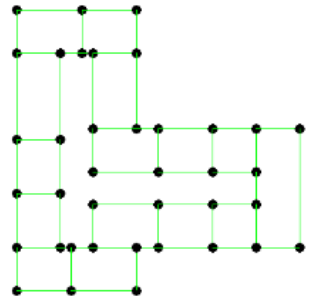

(b)

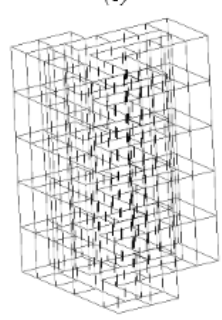

(d)
Figure 6. 3D building modeling and network analysis (a) Floor plan of building (b) Obtaining points and lines (c) Designing the floors (d) 3D Building Model (Karas et al., 2006).

The Architecture, engineering and construction (AEC) research communities intend to decrease cost and time of their project and to increase their quality. BIM is the most recent development of AEC. BIM is used for route planning in emergency response. BIM integrates geometrical and semantic information and it can be integrated to Virtual Reality (VR) techniques (Ruppel et al., 2010). Vanclooster and Maeyer (2012) suggested that a proper indoor route planning requires indoor network, semantic information and connection between indoor and outdoor network which the integration of GIS and BIM can be a solution.

BIM provides indoor spatial information but it lacks spatial functionality (Daum and Borrmann, 2014). GIS provides spatial information relevant to outdoor, thus the integration of BIM and GIS can be used for emergency response (Teo and Cho, 2016).

Chen and Feng (2009) presented that for a smart city application, integration of BIM and GIS for emergency response is a priority. BIM has been used for safety analysis (Zhang and $\mathrm{Hu}, 2011$ ) and fire safety management (Ruppel and Schntz, 2011; Li et al., 2014). Ruppel et al. (2010) presented a system to find shortest path for 
emergency rescuers in a complex building structure. Generation of BIM models is labour-intensive, time consuming and with complex data structure.

Indoor navigation network models including GNM, Navigable Space Model, sub-division model and regulargrid model lack indoor data sources and abstraction methods. GNM models are mostly extracted from 2D plans and the extracted indoor network information is coarse. GNM models do not contain accurate indoor information and they are complex and time consuming to generate (Teo and Cho, 2016).

\section{DATA COLLECTION AND 3D BUILDING MODELING}

For many kinds of systems like disaster or emergency management systems, the interior models are essential. Indoor models can be reconstructed from construction plans, but sometimes they are not available or very often they differs from 'as-built' plans. In this case the buildings and its rooms must be surveyed. The most utilized method of indoor surveying is laser scanning (Dongzhen et al., 2009; Yusuf, 2007). This method allows taking accurate and detailed measurements. However, Terrestrial Laser Scanner is costly and time consuming.

In this paper, Trimble M3 as surveying instrument for 3D data acquisition is used (see Figure 7). To establish surveying benchmarks (control points), closed traverse surveying was used. Traverse surveying is a method of surveying for establishing control points along with traveling lines or movement paths. Closed traverse will start from one point with known coordinates and will end in the same point. Traverse surveying was employed due to its nature that could adapt to complicated shapes which in this research, complicated shapes (buildings) consideration is required. Considering a known point A (xA,yA), coordinates of an unknown point $\mathrm{B}(\mathrm{xB}, \mathrm{yB})$ is calculated (equation (1)).

$x_{B}=x_{A} \pm A B \sin G_{A B} y_{B}=y_{A} \pm A B \cos G_{A B}$

Where $\mathrm{AB}=$ distance between point $\mathrm{A}$ and $\mathrm{B}$

$G_{A B}=$ Angle of Length AB towards north direction
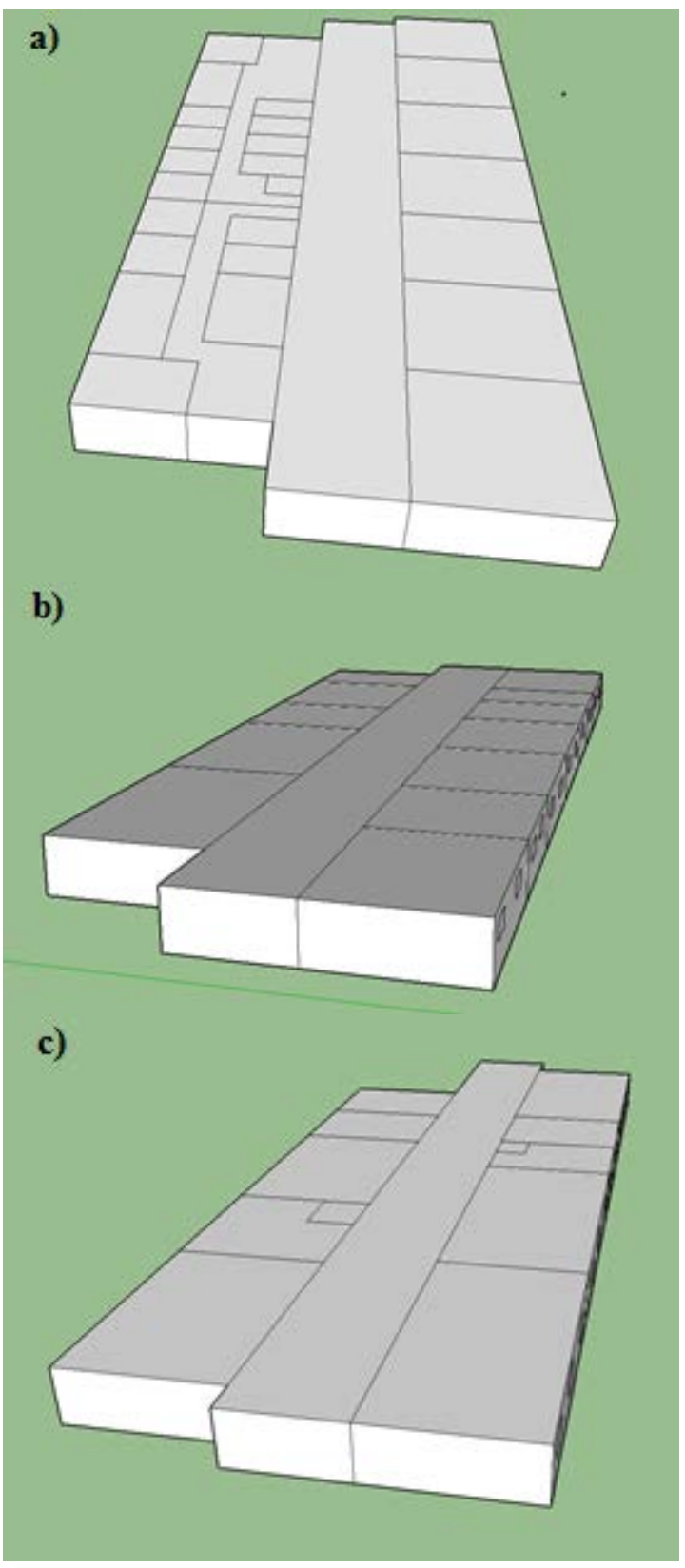

Figure 7. 3D building modeling using Trimble M3 a) first floor b) second floor c) third floor.

GIS integrates spatial information and spatial analysis. An important example of such integration is emergency response which requires route planning inside and outside of a building. Route planning requires detailed information related to indoor and outdoor environment. The DHE data structure was used to model data collected using Trimble M3 (See Figure 8). 


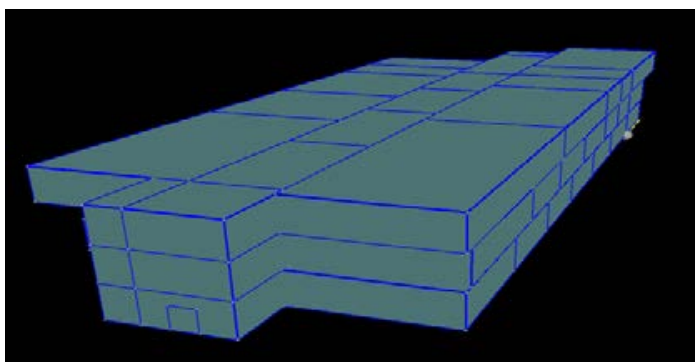

Figure 8. 3D building modeling using DHE data structure

DHE data structure is used for 3D spatial modeling. This structure resolves some of the modeling issues in 3D GIS and expresses the geometric structures as a cell complex, in preserving adjacency relationships between cells, and including semantic information using attributes (Boguslawski, 2011; Boguslawski et al., 2011). The DHE is based on the quad-edge and its extension- the augmented quad-edge (Ledoux and Gold, 2007). These structures allow construction of models and their duals at the same time. Dual space is used to connect cells in a complex and to navigate between them. Other data structures like the half-edge or winged-edge do not provide for management of the duality.

The construction process using the DHE is based on Euler operators which are used widely in CAD) systems for BRep modeling. They preserve topological consistency of the model on each step of a construction process. Euler operators were described by Baumgart (1975), Braid et al. (1980), Mäntylä (1988). 3D navigation in models is based on navigation described by Ledoux and Gold (2007) for the 3D Voronoi/Delaunay model. DHE data structure requires accurate geometrical models (cells should touch each other whiteout any gap and intersection). In DHE data structure dual nodes are generated automatically from geometrical models.

Each room is considered as a cell which several attributes can be defined for each cell. Each parcel is considered as a cell which has geometrical and topological information. In the concept of DHE data structure each cell will be treated as a primal model (parcel) which is connected to the other cells (parcels) using dual model concepts. Adjacencies between rooms are considered as an important aspect of this research.

Navigation between cells and queries like neighbourhood cells (neighbourhoods of an interested room) of an interested cell can be answered by dual concepts. Overlapping problem between cells (overlapping between ownerships) can be solved by defining proper tolerance for coordinates of nodes and optimizing accuracy of 3D models to an acceptable range suitable for cadastre. Error estimation was considered to create a precise and consistent 3D model. Final 3D model consists of valid cells (geometry) which are connected properly (topology) modelled by DHE data structure with proper associated attributes.

\section{NAVIGATION NETWORK}

For collecting the required navigation network outside the building, OSM is used. OSM is a collaborative project which was begun in England in 2004 by Steve Coast. The task goes for making and giving free geographic information. OSM's information is distributed under the permit "Creative Commons Attribution-Share Alike 2.0 license" (Girres and Touya, 2010). Navigation network provided by OSM is not complete and it has several errors (e.g. some lines which represent road network are not connected) which require to be modified. OSM provides 2D plan of UTM campus building and navigation network (see Figure 9).

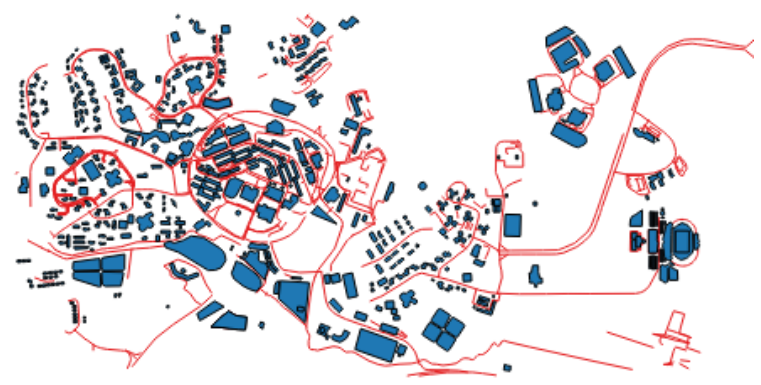

Figure 9. UTM buildings integrated with road network.

Data provided by OSM is $2 \mathrm{D}$ (i.e. road network and 2D building plan) and it requires several validation tests which will be next phase. Required elevation information is from ASTER Global DEM. ASTER GDEM is a product of METI and NASA (see Figure 10).

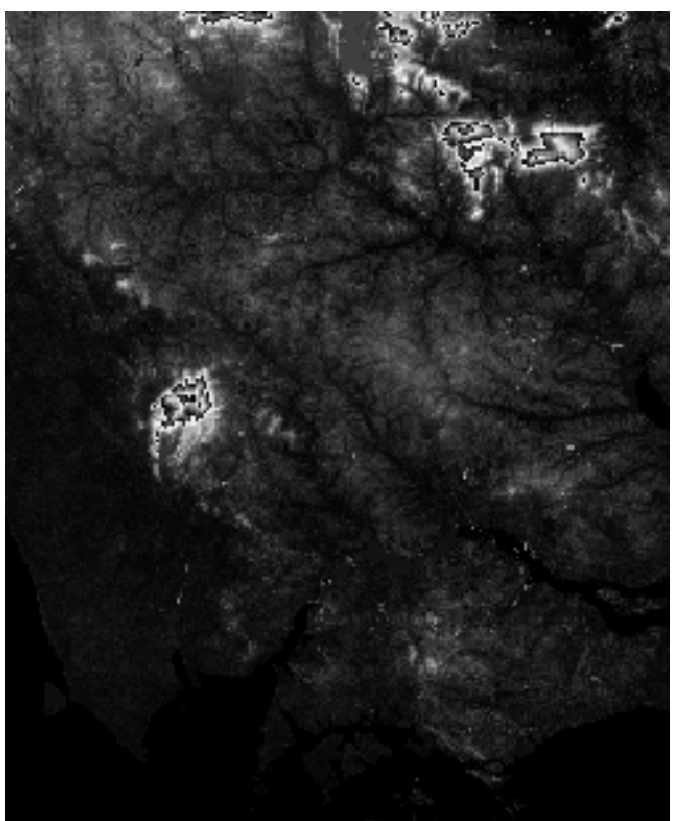

Figure 10. Elevation information from ASTER Global DEM. 
An integration of indoor building, outdoor building and navigation network is presented (see Figure 11).
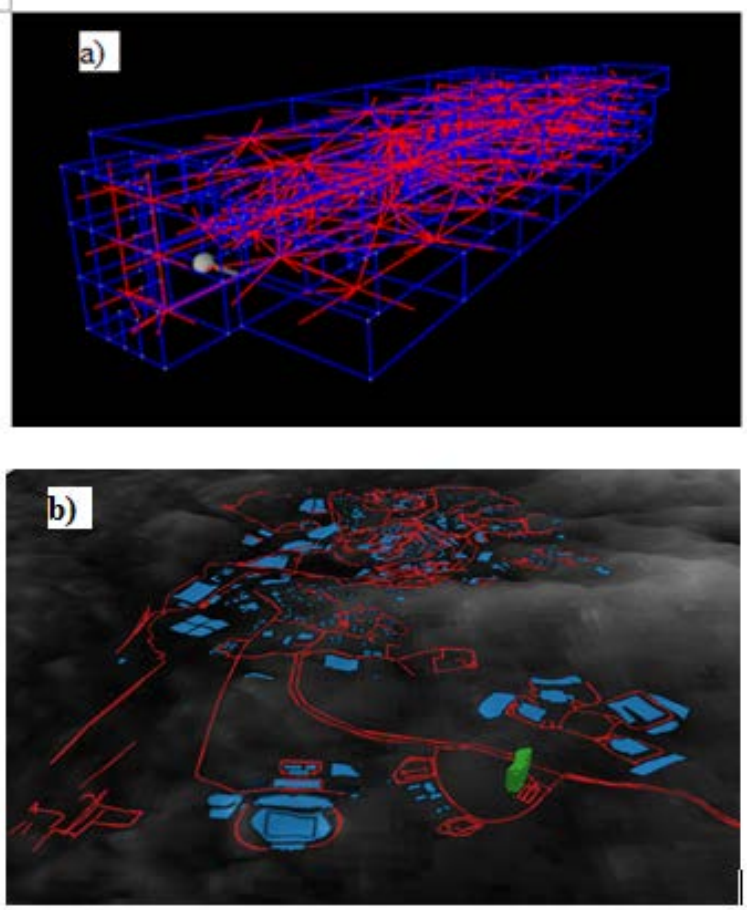

Figure 11. Indoor and Outdoor building modeling a) indoor building model b) outdoor building model (green colored building) integrated with navigation network and DEM.

At the moment of this research, indoor building model and outdoor navigation network integrated with DEM are analyzed separately. There is no information related to height of other buildings inside UTM campus.

\section{CONCLUSION}

In this paper, an integration of indoor building model, outdoor navigation network and DEM is presented. Trimble M3 was used to acquire data of indoor building environment. DHE data structure is used for 3D building modeling. Navigation network information outside of the building was collected by OSM. 3D Data storage and 3D data modeling need to be considered as most important factors in most of applications (e.g. 3D city modeling or disaster management systems). Keep in mind, navigation through a building is possible by nodes and lines (paths or edges) and 3D building models are for a better understanding of the real world environment (there is no need for high detailed 3D building models for navigation applications such as disaster management systems or for safety analysis). Data storage for three floors collected by Trimble M3 is 50 Kilo Bytes (KB) in DHE data structure. For a big dataset such as 3D models of a city, data storage is important.

\section{REFERENCES}

Bandi, S., \& Thalmann, D. (1998, August). Space discretization for efficient human navigation. In Computer Graphics Forum (Vol. 17, No. 3, pp. 195-206). Blackwell Publishers Ltd.

Baumgart, B., G., 1975. A polyhedron representation for computer vision, National Computer Conference and Exposition. ACM, Anaheim, California.

Becker, T., Nagel, C., \& Kolbe, T. H. (2009). A multilayered space-event model for navigation in indoor spaces. In 3D Geo-Information Sciences (pp. 61-77). Springer Berlin Heidelberg.

Boguslawski, P., Gold, C.M. and Ledoux, H., 2011. Modeling and analysing 3D buildings with a primal/dual data structure. ISPRS Journal of Photogrammetry and Remote Sensing, 66(2): 188-197.

Boguslawski, P., 2011. Modeling and Analysing 3D Building Interiors with the Dual Half-Edge Data Structure. PhD Thesis, University of Glamorgan, Pontypridd, Wales, UK, 134 pp.

Braid, I.C., Hillyard, R.C. and Stroud, I.A., 1980. Stepwise Construction of Polyhedra in Geometric Modeling. In: e. K.W.Brodlie (Editor), Mathematical Methods in Computer Graphics and Design. Academic Press, pp. 123-141.

Chen, P. H., \& Feng, F. (2009). A fast flow control algorithm for real-time emergency evacuation in large indoor areas. Fire Safety Journal, 44(5), 732-740.

Daum, S., \& Borrmann, A. (2014). Processing of topological BIM queries using boundary representation based methods. Advanced Engineering Informatics, 28(4), 272-286.

Dongzhen, J., Khoon, T., Zheng, Z., \& Qi, Z. (2009). Indoor 3D Modeling and Visualization with a 3D Terrestrial Laser Scanner. 3D Geo-Information Sciences, 247-255.

Donath, D., \& Thurow, T. (2007). Integrated architectural surveying and planning. Automation in Construction, 16(1), 19-27.

Girres, J. F., \& Touya, G. (2010). Quality assessment of the French OpenStreetMap dataset. Transactions in GIS, 14(4), 435-459.

Jamali, A., Abdul Rahman, A., Boguslawski, P., Kumar, P., \& M. Gold, C. (2015). An automated 3D modeling of topological indoor navigation network. GeoJournal.

Karas, I. R., Batuk, F., Akay, A. E., \& Baz, I. (2006). Automatically extracting 3D models and network analysis for indoors. In Innovations in 3D Geo Information Systems (pp. 395-404). Springer Berlin Heidelberg. 
Ledoux, H. and Gold, C.M., 2007. Simultaneous storage of primal and dual three-dimensional subdivisions. Computers, Environment and Urban Systems, 31(4): 393408.

Lee, J. (2001). 3D data model for representing topological relations of urban features. In Proceedings of the 21st Annual ESRI International User Conference, San Diego, CA, USA.

Lee, J. (2004). A spatial access-oriented implementation of a 3-D GIS topological data model for urban entities. GeoInformatica, 8(3), 237-264.

Lee, J., \& Kwan, M. P. (2005). A combinatorial data model for representing topological relations among 3D geographical features in micro-spatial environments. International Journal of Geographical Information Science, 19(10), 1039-1056.

Li, N., Becerik-Gerber, B., Krishnamachari, B., \& Soibelman, L. (2014). A BIM centered indoor localization algorithm to support building fire emergency response operations. Automation in Construction, 42, 78-89.

Li, Y., \& He, Z. (2008). 3D indoor navigation: a framework of combining BIM with 3D GIS. In 44th ISOCARP congress.

Liu, L., \& Zlatanova, S. (2011, May). A" door-to-door" Path-finding Approach for Indoor Navigation. In Proceedings Gi4DM 2011: GeoInformation for Disaster Management, Antalya, Turkey, 3-8 May 2011. International Society for Photogrammetry and Remote Sensing (ISPRS).

Liu, L., \& Zlatanova, S. (2011). Towards a 3D network model for indoor navigation. Urban and Regional Data Management, UDMS Annual, 79-92.

Liu, L., \& Zlatanova, S. (2013). A Two-level Path-finding Strategy for Indoor Navigation. In Intelligent Systems for Crisis Management (pp. 31-42). Springer Berlin Heidelberg.

Lorenz, B., Ohlbach, H. J., \& Stoffel, E. P. (2006). A hybrid spatial model for representing indoor environments. In Web and Wireless Geographical Information Systems (pp. 102-112). Springer Berlin Heidelberg.

Schaap, J., Zlatanova, S., \& van Oosterom, P. J. M. (2011). Towards a 3D geo-data model to support pedestrian routing in multimodal public transport travel advices. Urban and Regional Data Management, UDMS Annual, 63-78.

Slingsby, A., \& Raper, J. (2008). Navigable space in 3D city models for pedestrians. In Advances in $3 D$ Geoinformation Systems (pp. 49-64). Springer Berlin Heidelberg.

Stoffel, E. P., Lorenz, B., \& Ohlbach, H. J. (2007). Towards a semantic spatial model for pedestrian indoor navigation. In Advances in Conceptual Modeling-
Foundations and Applications (pp. 328-337). Springer Berlin Heidelberg.

Tashakkori, H., Rajabifard, A., \& Kalantari, M. (2015). A new 3D indoor/outdoor spatial model for indoor emergency response facilitation. Building and Environment, 89, 170182.

Teo, T. A., \& Cho, K. H. (2016). BIM-oriented indoor network model for indoor and outdoor combined route planning. Advanced Engineering Informatics, 30(3), 268282.

Vanclooster, A., \& De Maeyer, P. (2012). Combining indoor and outdoor navigation: the current approach of route planners. In Advances in Location-Based Services (pp. 283-303). Springer Berlin Heidelberg.

Yuan, W., \& Schneider, M. (2010, November). Supporting $3 \mathrm{D}$ route planning in indoor space based on the LEGO representation. In Proceedings of the 2nd ACM SIGSPATIAL International Workshop on Indoor Spatial Awareness (pp. 16-23). ACM.

Yuan, W., \& Schneider, M. (2010). iNav: An indoor navigation model supporting length-dependent optimal routing. In Geospatial Thinking (pp. 299-313). Springer Berlin Heidelberg.

Yusuf, A., 2007. An approach for real world data modeling with the 3D terrestrial laser scanner for built environment. Automation in Construction, 16(6): 816-829.

Zhang, J. P., \& Hu, Z. Z. (2011). BIM-and 4D-based integrated solution of analysis and management for conflicts and structural safety problems during construction: 1. Principles and methodologies. Automation in construction, 20(2), 155-166.

Zlatanova, S., \& Baharin, S. S. K. (2008). Optimal navigation of first responders using DBMS. In 3rd International Conference on Information Systems for Crisis Response and Management 4th International Symposium on GeoInformation for Disaster Management (pp. 541-54). 